\title{
Early Estimation of Renal Function After Transplantation to Enable Appropriate Dosing of Critical Drugs: Retrospective Analysis of 103 Patients in a Single Center
}

\author{
Tobias T. Pieters ${ }^{1} \cdot$ Paul Beele $^{1} \cdot$ Arjan D. Van Zuilen $^{1} \cdot$ Marianne C. Verhaar $^{1} \cdot$ Alwin D. R. Huitema $^{2,3}$. \\ Maarten B. Rookmaaker ${ }^{1}$
}

Published online: 8 May 2020

(c) The Author(s) 2020

\begin{abstract}
Background Immediately after renal transplantation (RTX), estimation of renal function (eGFR) is important for drug dosing and the detection of potential complications. Conventional formulas cannot be used since the serum creatinine concentration is not at steady-state. In this study, we evaluated different dynamic renal function formulas (DRFFs) to estimate eGFR immediately after RTX.

Methods We retrospectively included 154 RTX patients, of whom 45 had delayed graft function (DGF) and required dialysis, and 6 had unstable graft function without the need for dialysis; 103 patients had early, and thereafter stable, graft function (EGF). DRFFs were evaluated to calculate eGFR 1 day after transplantation (T1) using a new dynamic creatinine clearance calculation (D3C), two previously published formulas (Jelliffe, and the kinetic eGFR [KeGFR]), and a naive predictor (Chronic Kidney Disease Epidemiology Collaboration [CKD-EPI] at T1). The estimated DRFF-based renal functions at T1 were compared with the CKD-EPI after stabilization of renal function 3 days after transplantation (eGFR-T3), which was considered the underlying renal function immediately after RTX.

Results The D3C showed low bias (mean prediction error [MPE] $-4.5 \mathrm{ml} / \mathrm{min} / 1.73 \mathrm{~m}^{2}$ ) and performed well on other outcome measures $\left(R^{2}=0.82\right.$, root mean squared error $[\mathrm{RMSE}]=11.8 \mathrm{ml} / \mathrm{min} / 1.73 \mathrm{~m}^{2}$, percentage of predictions within $30 \%$ of the reference value $\left[p_{30 \%}\right]=76 \%$ ). In addition, the D3C outperformed the KeGFR (MPE $20.5 \mathrm{ml} / \mathrm{min} / 1.73 \mathrm{~m}^{2}, R^{2}=0.79$, RMSE $\left.=26.9 \mathrm{ml} / \mathrm{min} / 1.73 \mathrm{~m}^{2}, p_{30 \%}=29 \%\right)$, Jelliffe $\left(\right.$ MPE $-13.3 \mathrm{ml} / \mathrm{min} / 1.73 \mathrm{~m}^{2}, R^{2}=0.76, \mathrm{RMSE}=19.1 \mathrm{ml} / \mathrm{min} / 1.73 \mathrm{~m}^{2}$, $p_{30 \%}=53 \%$ ), and the naive predictor (bias $-24.8 \mathrm{ml} / \mathrm{min} / 1.73 \mathrm{~m}^{2}, R^{2}=0.60, \mathrm{RMSE}=30.2 \mathrm{ml} / \mathrm{min} / 1.73 \mathrm{~m}^{2}, p_{30 \%}=21 \%$ ).

Conclusions The newly developed D3C enables reliable assessment of renal function immediately after RTX, provides crucial information for drug dosing, and might also advance the detection of functional decline, potentially improving treatment and renal outcome.
\end{abstract}

Tobias T. Pieters and Paul Beele contributed equally to this work.

Electronic supplementary material The online version of this article (https://doi.org/10.1007/s40262-020-00893-z) contains supplementary material, which is available to authorized users.

Maarten B. Rookmaaker

m.rookmaaker@umcutrecht.nl

1 Department of Nephrology and Hypertension, UMC Utrecht, Utrecht University, Heidelberglaan 100, 3584CX Utrecht, The Netherlands

2 Department of Clinical Pharmacy, UMC Utrecht, Utrecht University, Heidelberglaan 100, 3584CX Utrecht, The Netherlands

3 Department of Pharmacy and Pharmacology, Netherlands Cancer Institute, Plesmanlaan 121, 1066 CX Amsterdam, The Netherlands

\section{Introduction}

Renal transplantation (RTX) is the best treatment for patients with end-stage renal disease. After successful RTX, serum creatinine concentrations decrease swiftly due to the immediate function of the donor kidney. Assessment of renal function immediately after RTX is important for dosing of drugs that are eliminated by the kidney, including many antiviral, antibiotic, and antidiabetic drugs frequently used by these patients. A reliable early estimate of renal function is of importance to guide drug dosing and reduce complications.

Glomerular filtration rate is usually estimated (eGFR) by formulas such as the Chronic Kidney Disease Epidemiology Collaboration (CKD-EPI) and Modification of Diet in 


\section{Key Points}

Estimated glomerular filtration rate (eGFR) measurements during nonsteady-state creatinine are unreliable, while appropriate estimates may be important for drug dosing.

This study focuses on the accuracy and precision of existing dynamic renal function formulas (DRFFs) and a newly developed formula in a population of renal transplant patients.

In this study, we show that a newly developed DRFF (which requires sex, age, and twice-measured creatinine) is highly accurate and precise in estimating underlying renal function, and outperforms currently available alternatives.

Renal Disease (MDRD) [1, 2]. When serum creatinine is not in a steady state, estimation of the GFR using conventional formulas is unreliable and lags behind the true underlying GFR. Due to the rapid decrease in creatinine concentration after RTX, conventional formulas do not accurately estimate GFR [3-7].

Several dynamic renal function formulas (DRFFs) have been developed to estimate renal function during acute fluctuations of serum creatinine concentration [4-7]. These DRFFs are based on the pharmacokinetic principles of creatinine mass balance; when creatinine production exceeds excretion, creatinine will build up in the volume of distribution until excretion again equals production and a new steady state has been reached, or vice versa. Clearance can therefore be calculated using estimates of the volume of distribution and creatinine production combined with the observed change in serum creatinine over time. Different DRFFs utilize different estimations for volume of distribution and creatinine production, the accuracy of which may vary between different patient populations [8].

Although DRFFs have been evaluated in acute kidney injury in the intensive care unit (ICU), the experience with DRFFs in RTX is scarce [9-11]. In addition, the accuracy of these methods to estimate underlying GFR has not been investigated in any population, which is crucial if it is to be used for drug dosing. In addition, different DRFFs have not been evaluated side-by-side in the same study or population.

In this study, we developed and evaluated a new formula, the Dynamic Creatinine Clearance Calculation (D3C), which uses pharmacokinetic principles of creatinine mass balance and readily available variables (age, sex, and two successive creatinine concentrations) to estimate normalized GFR $\left(\mathrm{ml} / \mathrm{min} / 1.73 \mathrm{~m}^{2}\right)$ when creatinine is not in steady state, and compared its performance with two existing DRFFs, in a cohort of patients 1 day after RTX. We used eGFR at steady state creatinine 3 days after transplantation as the reference standard. The aim of this study was to assess and compare the accuracy and precision of different DRFFs to estimate underlying GFR in the first days after transplantation in order to optimize drug dosing in this crucial period immediately after transplantation.

\section{Methods}

\subsection{Patient Population}

We retrospectively included all 154 patients aged over 18 years who underwent RTX at the UMC Utrecht between January 2014 and July 2016. We excluded 45 patients who had delayed graft function (DGF) and required dialysis, since our analyses required patients to have immediate function after transplantation until they reached steady-state creatinine. Of those patients with early graft function (EGF), we excluded an additional six patients with immediate but unstable graft function, defined as a daily increase of more than $20 \%$ (normal analytical/biological variation) in serum creatinine in the analysis period (T0B-T3).

Patient data were systematically recorded in a renal transplant database during hospital admission until discharge. From this database, we obtained data on donor, recipient, procedure, and patient follow-up. For the recipient, we collected data on age, sex, dialysis modality, diabetes status, and height and weight on the day of transplantation, while for the donor, we collected data on age, sex, cold ischemia time, type of donor (living, donation after brain death, or donation after cardiac death), human leukocyte antigen (HLA) mismatches, weight, and last known serum creatinine level. Serum creatinine was determined by enzymatic colorimetric assay (Beckman Coulter, Brea, CA, USA).

DGF was defined as the need for dialysis within the first week after transplantation [12]. Patients with stable graft function were defined as patients with EGF without a daily $20 \%$ rise in creatinine, since this exceeds the biological and analytical variation of serum creatinine [13].

\subsection{Construction and Rationale of Dynamic Renal Function Formulas (DRFFs)}

All DRFFs have been constructed based on the mass balance equation in a single compartment model, given that the volume of distribution remains stable (Eq. 1) [14]:

$\mathrm{CLcr}=\frac{\mathrm{Pcr}-\mathrm{Vd} \times \frac{\mathrm{d} C}{\mathrm{~d} T}}{C}$ 
where CLcr is the clearance, Pcr is the production of creatinine, $\mathrm{Vd}$ is the volume of distribution, $T$ represents a unit of time, and $C$ is the creatinine concentration. One can simplify the above equation by substituting the differential $\frac{\mathrm{d} C}{\mathrm{~d} T}$ in the numerator by $\frac{\Delta C}{\Delta T}$ of two subsequent creatinine measurements, and $C$ in the denominator by the mean of these two measurements.

\subsection{Dynamic Creatinine Clearance Calculation (D3C)}

For the D3C, we estimated mean creatinine with the geometric mean, which is consistent with the proposed first-order elimination of creatinine. D3C creatinine clearance (D3C*) can therefore be calculated using the following formula (Eq. 2):

$\mathrm{D} 3 \mathrm{C} *=\frac{\mathrm{Pcr}-\mathrm{Vd} \times \frac{\Delta C}{\Delta T}}{\text { Cgeomean }}$,

where Cgeomean is the geometric mean creatinine.

The volume of distribution of creatinine has been shown to equal total body water, which, after RTX, has been shown to be approximately $0.6 \mathrm{~L} / \mathrm{kg}$ of bodyweight [15-17]. Production of creatinine was derived from the Cockcroft-Gault formula (Eq. 3) [18]:

$\operatorname{Pcr}(\mu \mathrm{mol} / \mathrm{h})=(140-A) \times W \times 0.07362[\times 0.85$ if female $]$,

where $A$ represents age (years) and $W$ represents bodyweight $(\mathrm{kg})$.

The D3C was rewritten to give an eGFR instead of an estimated creatinine clearance $(\mathrm{eCrCl})$. We did this by calculating projected steady-state creatinine (Eqs. 4, 5):

$\frac{\mathrm{Pcr}-\mathrm{Vd} \times \frac{\Delta C}{\Delta T}}{\text { Cgeomean }}=\frac{\mathrm{Pcr}}{\mathrm{Css}}$,

$$
\begin{aligned}
& \frac{(140-A) \times W \times 0.07362[\times 0.85 \text { if } F]-0.6 \times W \times \frac{\Delta C}{\Delta T}}{\text { Cgeomean }} \\
& =\frac{(140-A) \times W \times 0.07362[\times 0.85 \text { if } F]}{\operatorname{Css}},
\end{aligned}
$$

After rearranging, this becomes (Eqs. 6, 7):

Cssmale $(\mu \mathrm{Mol} / \mathrm{L})=\frac{\text { Cgeomean } \times(140-A) \times 0.07362}{0.07362 \times(140-A)-0.6 \times \frac{\Delta C}{\Delta T}}$,

Cssfemale $(\mu \mathrm{Mol} / \mathrm{L})=\frac{\text { Cgeomean } \times(140-A) \times 0.06258}{0.06258 \times(140-A)-0.6 \times \frac{\Delta C}{\Delta T}}$,

where Cgeomean is the geometric mean creatinine $(\mu \mathrm{mol} / \mathrm{l})$, $A$ represents age (years), $\Delta C$ is the change in creatinine concentration $(\mu \mathrm{Mol} / \mathrm{l})$, and $\Delta T$ represents the change in time (h).

Since weight can be eliminated in the above rearrangement, the calculated steady-state creatinine can be calculated without knowing the patient's weight, and can be incorporated into the standard CKD-EPI formula to give the $\mathrm{D} 3 \mathrm{C}$ as an eGFR in $\mathrm{ml} / \mathrm{min} / 1.73 \mathrm{~m}^{2}$ [2].

\subsection{Kinetic Estimated Glomerular Filtration Rate}

The kinetic eGFR (KeGFR) estimates volume of distribution by dividing the total body change in creatinine by the change in plasma creatinine when a patient is anuric, when change in total body creatinine equals the production of creatinine (Eqs. 8, 9):

$\mathrm{Vd}=\frac{\Delta C \text { tot }}{\Delta C}$,

$\mathrm{Vd}=\frac{\text { Pcr }}{\text { Max } \Delta C / \text { day when GFR }=0}$.

Production of creatinine is estimated by multiplying any estimate of eGFR or eCrCl with a coupled known steadystate creatinine concentration, or, when this is unknown, the Cockcroft-Gault formula. After dividing the total by the arithmetic mean, and after rearrangement, the formula is (Eq. 10):

KeGFR $=\frac{\text { Css } \times \text { eGFR }}{\text { Carrmean }} \times\left(1-\frac{24 \times \Delta C}{\Delta t \times \operatorname{Max} \Delta C / \text { day }}\right)$.

Since the last known steady-state creatinine and renal function could not be derived for patients who had been on dialysis before RTX, we used the Cockcroft-Gault formula [5]. In addition, since we did not have records detailing whether our patients had been fully anuric in the past, we used the default value of $132.6 \mu \mathrm{Mol} / \mathrm{l} /$ day suggested by the author.

\subsection{Jelliffe Calculations}

Jelliffe estimates volume of distribution (dL) by multiplying body weight by 4 . Production of creatinine ( $\mathrm{mg} / \mathrm{day})$ is estimated as follows (Eq. 11):

$[29.305-(0.203 \times A)] \times W \times[1.037-(0.0338 \times$ Carrmean $)]$

\subsection{Evaluation of DRFFs}

Creatinine was sampled at different time points: the recovery unit (T0), when the patient reached the nursing ward (T0B, $4.2 \pm 0.9 \mathrm{~h}$ ), and every morning at the nursing ward (T1-T6). 
We did not use the T0-TB interval to calculate the DRFFs, since the time between measurements at the T0-TB interval is small and the time annotation of samples collected at the recovery unit might not be fully accurate. Furthermore, it is likely that patients with underlying renal function in the first hours after transplantation are still recovering from the effects of the surgery, thus renal function at this stage will not coincide with renal function at $\mathrm{T} 3$. Therefore, the T0B-T1 interval $(11.3 \pm 2.8 \mathrm{~h})$ was used and compared with eGFR (CKD-EPI) 3 days after transplantation, the earliest point at which creatinine, on average, reached steady-state, defined as the moment when the average change in serum creatinine approximated $0 \%$ (T3, Fig. 1b). For this analysis,

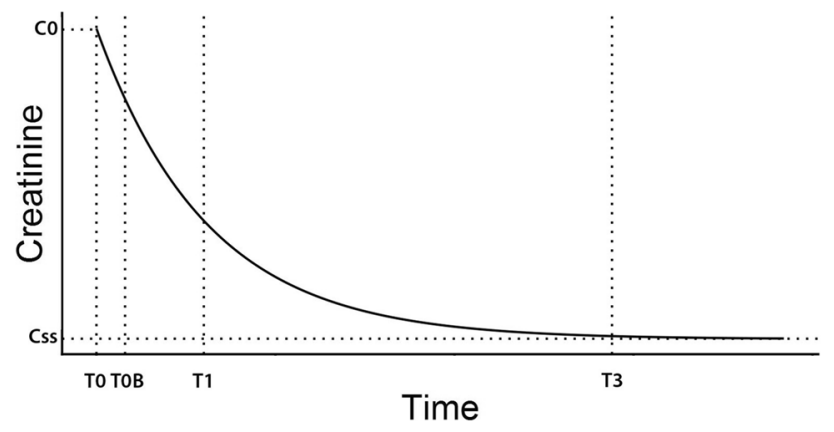

Fig. 1 Modeled example of serum creatinine course in a patient after transplantation, and sampling time points: T0 is sampled when patients enter the recovery unit; T0B is sampled when patients reach the nursing ward; T1 is sampled the next day during the morning round; and $\mathrm{T} 3$ is sampled 3 days after transplantation during the morning round when creatinine has reached steady-state. Since the time between measurements at the T0-TB interval is small (mean $4 \mathrm{~h}$ ) and the time annotation of samples collected at the recovery unit might not be fully accurate, the T0B-T1 interval (mean $16 \mathrm{~h}$ ) was used to calculate the dynamic renal function and compare with steady-state eGFR at T3. $\mathrm{CO}$ creatinine concentration in the recovery unit, Css steady-state creatinine concentration, eGFR estimated glomerular filtration rate only patients with EGF were included, since creatinine is not an accurate measure of renal function when patients are on dialysis $[12,14,19]$. In patients with EGF, we assumed underlying renal function to be constant between $\mathrm{T} 1$ and $\mathrm{T} 3$ in patients who are not suspected of having significant functional decline, defined as a $20 \%$ increase in serum creatinine concentration [13].

The performance of the $\mathrm{D} 3 \mathrm{C}$ was compared with a naive predictor (CKD-EPI at T1) and previous approaches to estimate renal function in case of nonsteady-state serum creatinine concentrations, i.e. the KeGFR described by Chen and Jelliffe's formula of creatinine clearance $[5,6]$. The input parameters of the formulas are summarized in Table 1. Performance of the different formulas at $\mathrm{T} 1$ was tested by assessing the mean prediction error (MPE), $R^{2}$, root mean squared error (RMSE), and the percentage of predictions within $30 \%$ of the reference value $\left(p_{30 \%}\right)$. To allow comparison with the CKD-EPI, the Jelliffe and KeGFR formulas were indexed to a body surface area (BSA) of $1.73 \mathrm{~m}^{2}$ using the Du Bois formula [20].

\subsection{Urinary Creatinine Clearance}

In order to further test the validity of the dynamic formulas, we calculated urinary creatinine clearance in a subset of patients $(n=83)$ for whom 24-h urine was collected the day after transplantation. We calculated urinary creatinine clearance as follows (Eq. 12):

$\mathrm{uCrCl}=\frac{\mathrm{UCr} \times \mathrm{Uvol}}{1.44 \times \text { Cgeomean }}$,

where $\mathrm{uCrCl}$ is the creatinine clearance $(\mathrm{ml} / \mathrm{min}), \mathrm{UCr}$ is the urinary creatinine concentration $(\mathrm{mmol} / \mathrm{l}), \mathrm{Uvol}$ is the urinary volume $(\mathrm{ml})$, and Cgeomean is the geometric mean

Table 1 Calculation of pharmacokinetic parameters of creatinine

\begin{tabular}{|c|c|c|c|c|c|c|}
\hline & \multicolumn{2}{|l|}{$\mathrm{D} 3 \mathrm{C}$} & \multicolumn{2}{|l|}{ KeGFR } & \multicolumn{2}{|l|}{ Jelliffe } \\
\hline & Method & Value $^{\mathrm{a}}$ & Method & Value $^{\mathrm{a}}$ & Method & Value $^{\mathrm{a}}$ \\
\hline Production $(\mu \mathrm{Mol} / \mathrm{h})$ & Cockcroft-Gault & $470 \pm 120$ & Cockcroft-Gault & $470 \pm 120$ & Jelliffe & $450 \pm 100$ \\
\hline Volume of distribution (1) & $60 \%$ of $\mathrm{BW}^{\mathrm{b}}$ & $46.0 \pm 9.1$ & $\begin{array}{l}\text { Creatinine } \\
\text { increase during } \\
\text { anuria }^{c}\end{array}$ & $59.8 \pm 15.2$ & $40 \%$ of $\mathrm{BW}$ & $30.7 \pm 6.1$ \\
\hline Mean creatinine value $(\mu \mathrm{Mol} / \mathrm{l})$ & Geometric mean & $398[81-1485]^{\mathrm{d}}$ & Arithmetic mean & $416[88-1485]^{\mathrm{c}}$ & Arithmetic mean & $416[88-1485]^{\mathrm{C}}$ \\
\hline
\end{tabular}

$D 3 C$ dynamic creatinine clearance calculation, $K e G F R$ kinetic estimated glomerular filtration rate, $B W$ bodyweight, $S D$ standard deviation, $V d$ volume of distribution

${ }^{\mathrm{a}}$ Data are expressed as mean $\pm \mathrm{SD}$ or median [range]

${ }^{\mathrm{b}} \mathrm{BW}$ is eventually eliminated in the $\mathrm{D} 3 \mathrm{C}$ formula and is therefore not needed for calculations

${ }^{\mathrm{c}} \mathrm{Vd}=\frac{\text { Pcreat }}{\Delta C / \Delta T \text { when GFR }=0}$; if anuric serum creatinine increase is unknown, default is $132.6 \mu \mathrm{Mol} / \mathrm{day}$

${ }^{\mathrm{d}}$ Average geometric mean at 1 day after transplantation (T1) 
of serum creatinine concentration from the beginning and end of collection $(\mu \mathrm{mol} / \mathrm{l})$.

In our center, urine collection is measured and sent to the laboratory at $2200 \mathrm{~h}$. Since we did not have the serum creatinine concentrations at the beginning and start of the urine collection, of which we would normally take the geometric mean, we used the serum creatinine concentration at $\mathrm{T} 1$ as the mean value. The $\mathrm{uCrCl}$ was normalized to $1.73 \mathrm{~m}^{2} \mathrm{BSA}$ using the Du Bois formula [20].

\subsection{Sensitivity Analysis}

To test the robustness of the $\mathrm{D} 3 \mathrm{C}$ to variable assumptions of the volume of distribution, we calculated the D3C with differing assumptions of volume of distribution, holding the other parameters constant (Table 5). To test how much of the performance of the $\mathrm{D} 3 \mathrm{C}$ to estimate eGFR 2 days later might be affected by normal fluctuation of the eGFR, we evaluated the RMSE of serial eGFR measurements during steady-state creatinine over 2 days (T3-T5).

\subsection{Statistics}

Normality of continuous variables was assessed using histograms. Missing values were analyzed via pairwise deletion; changes in creatinine over time were analyzed as log-change per hour; and differences in log-change per hour between different days were analyzed using the paired $t$ test. The $R^{2}$ value was acquired by ordinary least squares regression, and accuracy was acquired using Bland-Altman plots, the RMSE, and as the percentage of cases within a $30 \%$ range $\left(p_{30 \%}\right)$.

$p$ values $<0.05$ were considered statistically significant. All calculations and statistical analyses were executed using $\mathrm{R}$ statistics version 3.6.1.

\section{Results}

\subsection{Patient Characteristics}

We included 103 patients who underwent kidney transplantation in our center. The first creatinine measurement to evaluate underlying renal function was taken at $4.2 \pm 0.9 \mathrm{~h}$ after transplantation (T0B). In patients with EGF, there was a rapid decline of serum creatinine after transplantation, and, in the majority of patients, steady-state was reached at day 3 (T3), defined as a non-significant change in creatinine from T3 to T4 compared with T4-T5 ( $p=0.16$ ) (Fig. 1a, b). All patients received standard immunosuppressive therapy, consisting of prednisolone, tacrolimus, and mycophenolic acid. Population characteristics are shown in Table 2.
Daily calculation of DRFFs after transplantation showed that the D3C remained stable, the KeGFR showed a decline, and the Jelliffe formula showed a rise in renal function until steady-state creatinine (electronic supplementary Fig. 1). At steady state (T3), the D3C (mean difference $1.3 \mathrm{ml} /$ $\min / 1.73 \mathrm{~m}^{2}$ ) and Jelliffe (mean difference $-0.03 \mathrm{ml} /$ $\min / 1.73 \mathrm{~m}^{2}$ ) formulas coincided with the CKD-EPI, while the KeGFR had a higher estimate (mean difference $10.5 \mathrm{ml} /$ $\min / 1.73 \mathrm{~m}^{2}$ ). Table 3 shows the difference in classification of renal function categories between the D3C and the CKD-EPI at T1: $19 \%$ of patients did not change categories, however $35 \%$ changed one category and $46 \%$ changed more than one category.

\subsection{The D3C Accurately Quantifies the Estimated Glomerular Filtration Rate When Serum Creatinine is Not in Steady State}

To assess the ability of the formulas to predict underlying renal function directly after RTX, we compared DRFFs at T1 with CKD-EPI eGFR at steady-state (eGFR-T3) (Table 4). The D3C displayed the least bias in predicting eGFR-T3 (MPE $-4.5 \mathrm{ml} / \mathrm{min} / 1.73 \mathrm{~m}^{2}$ ). The overall tendency of the Jelliffe formula was to underestimate eGFRT3 (MPE - $13.3 \mathrm{ml} / \mathrm{min} / 1.73 \mathrm{~m}^{2}$ ), while the KeGFR formula overestimated eGFR-T3 (MPE $20.5 \mathrm{ml} / \mathrm{min} / 1.73 \mathrm{~m}^{2}$ ) (Table 4). The precision of all DRFFs was excellent (Table 4 , Fig. $2 \mathrm{c}-\mathrm{f})$. The D3C $\left(R^{2}=0.816, p<0.001\right)$ had slightly higher precision than the KeGFR $\left(R^{2}=0.787, p<0.001\right)$ and Jelliffe $\left(R^{2}=0.762, p<0.001\right)$ formulas. Finally, the D3C

Table 2 Characteristics of recipients and donors $(N=103)^{\mathrm{a}}$

\begin{tabular}{lll}
\hline Parameters & & \\
\hline Recipient & & \\
Age (years) & $50 \pm 15^{\mathrm{a}}$ & \\
Male/female & $67 / 36$ & \\
Weight (kg) & $77 \pm 15^{\mathrm{a}}$ & \\
Diabetes (yes/no) & $27 / 67$ & \\
Type of dialysis (HD/PD/pre-emptive) & $38 / 27 / 38$ & \\
Donor & Living & Post-mortal \\
Age (years) & $55 \pm 11^{\mathrm{a}}$ & $53 \pm 13^{\mathrm{a}}$ \\
Male/female & $25 / 46$ & $16 / 16$ \\
Living/DBD/DCD & $71 / 0 / 0$ & $0 / 18 / 14$ \\
Cold ischemia time (h) & $2 \pm 0.5^{\mathrm{a}}$ & $16 \pm 9^{\mathrm{a}}$ \\
HLA mismatch $(\leq 4 />4)$ & $44 / 27$ & $27 / 3$ \\
Weight $(\mathrm{kg})$ & $73 \pm 13^{\mathrm{a}}$ & $77 \pm 16^{\mathrm{a}}$ \\
Preoperative creatinine $(\mu \mathrm{mol} / \mathrm{ml})$ & $68 \pm 14^{\mathrm{a}}$ & $68 \pm 25^{\mathrm{a}}$ \\
\hline
\end{tabular}

$H D$ hemodialysis, $P D$ peritoneal dialysis, $D B D$ donation after brain death, $D C D$ donation after cardiac death, $H L A$ human leukocyte antigen, $S D$ standard deviation

${ }^{\mathrm{a}} \mathrm{Mean} \pm \mathrm{SD}$ 
Table 3 Comparison of D3C and the CKD-EPI formula at $\mathrm{T} 1$ in the eGFR stage

\begin{tabular}{|c|c|c|c|c|c|c|}
\hline \multirow{2}{*}{$\begin{array}{l}\text { KDIGO stage }(\mathrm{ml} / \\
\left.\mathrm{min} / 1.73 \mathrm{~m}^{2}\right)\end{array}$} & \multicolumn{6}{|c|}{ CKD-EPI } \\
\hline & $\geq 90$ & $60-89$ & $45-59$ & $30-44$ & $15-29$ & $<15$ \\
\hline \multicolumn{7}{|l|}{ D3C } \\
\hline$\geq 90$ & 1 & 1 & 0 & 0 & 0 & 0 \\
\hline $60-89$ & 0 & 1 & 4 & 6 & 6 & 1 \\
\hline $45-59$ & 0 & 0 & 0 & 5 & 20 & 0 \\
\hline $30-44$ & 0 & 0 & 0 & 0 & 14 & 14 \\
\hline $15-29$ & 0 & 0 & 0 & 0 & 2 & 12 \\
\hline$<15$ & 0 & 0 & 0 & 0 & 0 & 15 \\
\hline
\end{tabular}

$D 3 C$ dynamic creatinine clearance calculation, $C K D$-EPI Chronic Kidney Disease Epidemiology Collaboration, $e G F R$ estimated glomerular filtration rate, KDIGO Kidney Disease Improving Global Outcomes
Table 4 Comparison of the D3C with previously published formulas; analysis of bias, precision and accuracy compared with the CKD-EPI estimated steady-state renal function at T3 in patients with EGF and stable graft function $(N=103)$

\begin{tabular}{lllll}
\hline & Naive & D3C & KeGFR & Jelliffe \\
\hline $\begin{array}{l}\text { Estimated } \\
\text { underlying }\end{array}$ & $21.3 \pm 15.2$ & $41.9 \pm 22.3$ & $67.3 \pm 34.8$ & $33.0 \pm 16.6$ \\
$\quad$ eGFR $^{\mathrm{a}}$ & & & & \\
MPE & -24.8 & -4.5 & 20.5 & -13.3 \\
$R^{2 \mathrm{~b}}$ & 0.60 & 0.82 & 0.79 & 0.76 \\
RMSE & 30.2 & 11.8 & 26.9 & 19.3 \\
$p_{30 \%}{ }^{\mathrm{b}}$ & $21 \%$ & $76 \%$ & $29 \%$ & $53 \%$ \\
\hline
\end{tabular}

$D 3 C$ dynamic creatinine clearance calculation, CKD-EPI Chronic Kidney Disease Epidemiology Collaboration, eGFR estimated glomerular filtration rate, $E G F$ early graft function, $K e G F R$ KeGFR kinetic estimated glomerular filtration rate, $M P E$ mean prediction error, $S D$ standard deviation, $R M S E$ root mean squared error

${ }^{\mathrm{a}} \mathrm{Mean} \pm \mathrm{SD}$

${ }^{b}$ Goodness-of-fit, measure of estimation precision, derived from ordinary linear least squares regression

${ }^{c}$ Accuracy defined as the percentage of subjects within $30 \%$ of the CKD-EPI estimated renal function

had the lowest RMSE $\left(11.8 \mathrm{ml} / \mathrm{min} / 1.73 \mathrm{~m}^{2}\right)$ and highest $p_{30 \%}$ of the formulas, with $76 \%$ of the estimates within $\pm 30 \%$ of the eGFR-T3 (Table 4, Fig. 2g-j).

In addition, in a subset of patients $(n=83)$, we calculated the urinary creatinine clearance from the 24-h urine collected at the end of T1 (collected between T0 $2200 \mathrm{~h}$ and $\mathrm{T} 12200 \mathrm{~h}$ ) and compared this with the dynamic formulas at T1 (median time of measurement $0800 \mathrm{~h}$ ). For this purpose, we used the untransformed D3C (D3C*, calculated using Eq. 2) to calculate creatinine clearance instead of eGFR. Performance of the D3C* $(\mathrm{MPE}=-1.0 \mathrm{ml} /$ $\min / 1.73 \mathrm{~m}^{2}, R^{2}=0.76, \mathrm{RMSE}=13.0 \mathrm{ml} / \mathrm{min} / 1.73 \mathrm{~m}^{2}$, $\left.p_{30 \%}=74 \%\right)$, Jelliffe $\left(\mathrm{MPE}=-16 \mathrm{ml} / \mathrm{min} / 1.73 \mathrm{~m}^{2}\right.$, $R^{2}=0.73$, RMSE $\left.=22.5 \mathrm{ml} / \mathrm{min} / 1.73 \mathrm{~m}^{2}, p_{30 \%}=48 \%\right)$, and Chen formulas $\left(\mathrm{MPE}=19 \mathrm{ml} / \mathrm{min} / 1.73 \mathrm{~m}^{2}, R^{2}=0.68\right.$, $\mathrm{RMSE}=27.3 \mathrm{ml} / \mathrm{min} / 1.73 \mathrm{~m}^{2}, p_{30 \%}=37 \%$ ) were comparable with the analyses using the CKD-EPI at T3 (electronic supplementary Fig. 2).

\subsection{Sensitivity Analysis Reveals that the D3C is Robust to Assumptions in Volume of Distribution That is Subjected to latrogenic Fluctuation During Transplantation}

The assumption regarding volume of distribution might be violated. Patients with end-stage renal disease, especially those treated with hemodialysis, are often exposed to rapid and large changes in distribution volume. Moreover, during and immediately after transplantation, patients may receive aggressive volume reconstitution during or after transplantation, which in turn affects the performance of the D3C. Sensitivity analysis revealed that significant decreases $(50 \%$ of bodyweight, mean difference $-4.7 \pm 2.9 \mathrm{ml} / \mathrm{min} / 1.73 \mathrm{~m}^{2}$ ) or increases ( $70 \%$ of bodyweight, mean difference $4.7 \pm 2.9 \mathrm{ml} /$ $\mathrm{min} / 1.73 \mathrm{~m}^{2}$ ) in total body water composition of the patient did not significantly alter the D3C (Table 5). Additional subgroup analyses of the $\mathrm{D} 3 \mathrm{C}$ also showed that patients who were on peritoneal dialysis $(\mathrm{MPE}=-3.0, \mathrm{RMSE}=9.4)$ or hemodialysis $(\mathrm{MPE}=-5.5, \mathrm{RMSE}=12.1)$, or were preemptive $(\mathrm{MPE}=-4.0, \mathrm{RMSE}=13.0)$ prior to transplantation had similar bias and accuracy.

Furthermore, natural fluctuations in underlying GFR between T1 and T3 may introduce extra variability, leading to an artificial increase in prediction error. It is of note that after stabilization of creatinine concentration at day 3, the CKD-EPI fluctuated with an RMSE of $8.9 \mathrm{ml} / \mathrm{min} / 1.73 \mathrm{~m}^{2}$ over a 2-day period (T3-T5).

\section{Discussion}

Early assessment of renal function immediately after RTX is of critical importance to guide drug dosing and direct treatment decisions. Many drugs used early after transplantation, such as antivirals and antibiotics, are cleared renally and 

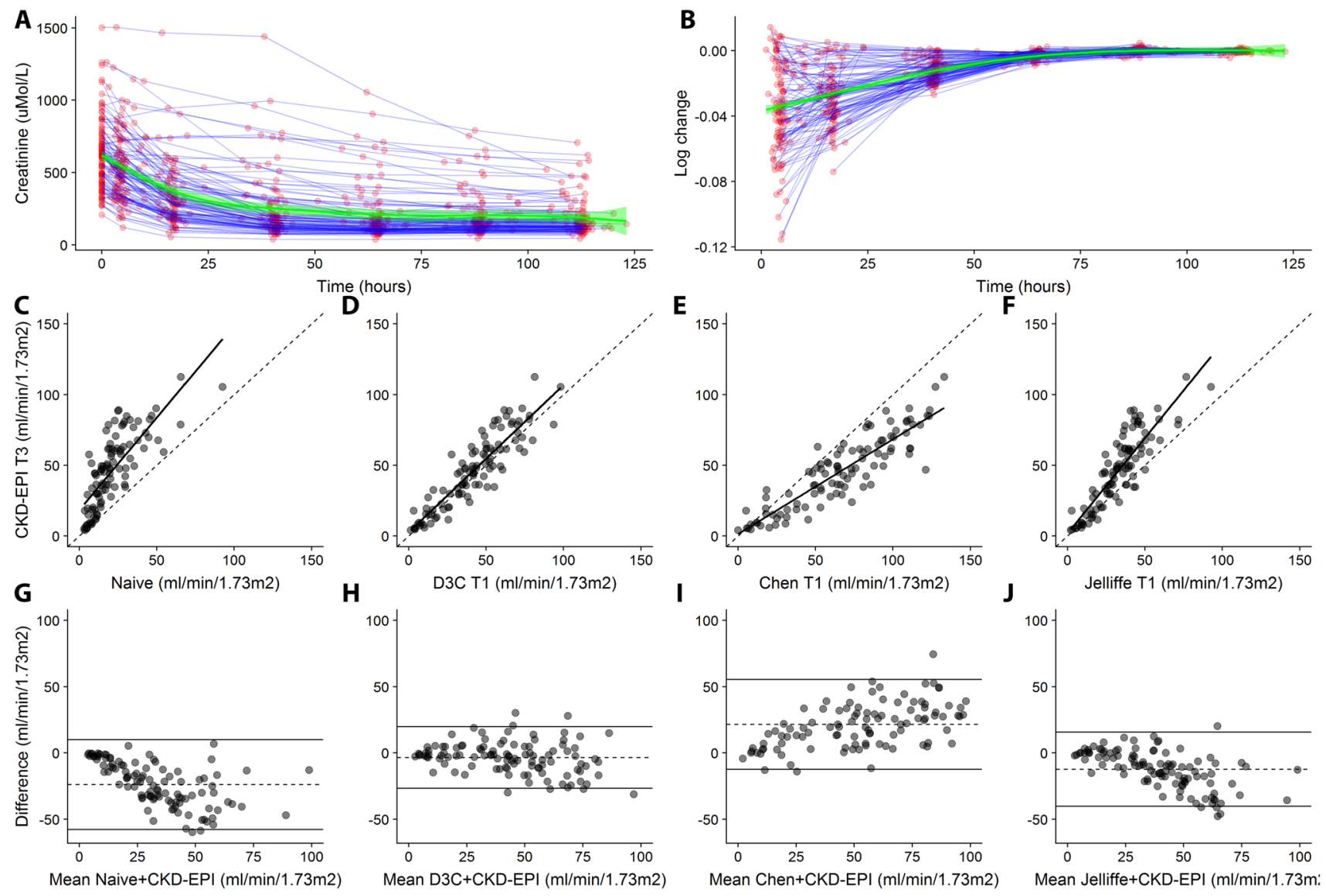

Fig. 2 D3C calculated the day after transplantation $(14.9 \pm 4.2 \mathrm{~h}$ after transplantation) is highly accurate in estimating the underlying renal function, defined as steady-state eGFR (CKD-EPI) 3 days after transplantation in patients with stable graft function. a, b Scatter plots showing the creatinine course (a) and log change per hour compared with the last measurement (b) over time in patients with EGF the first week after transplantation. Red dots represent the measurements, with blue lines connecting individual measurements per patient. The green line represents a smoothed LOESS line. c-f Scatter plots of all predictors 1 day after transplantation, and CKD-EPI-calculated eGFR 3 days after transplantation. The dotted line represents the line of

identity. The regression line (solid line) and $R^{2}$ values were calculated using the ordinary least squares regression line. g-j Bland-Altman plots of all predictors 1 day after transplantation, and CKD-EPI-calculated eGFR 3 days after transplantation. The dotted line represents the mean difference, and solid lines represent the mean difference $\pm 1.96 \mathrm{SD}$. $D 3 C$ dynamic creatinine clearance calculation, $e G F R$ estimated glomerular filtration rate, CKD-EPI Chronic Kidney Disease Epidemiology Collaboration, EGF early graft function, LOESS locally estimated scatterplot smoothing, $e G F R$ estimated glomerular filtration rate, $S D$ standard deviation

Table 5 Sensitivity analysis of different assumptions of the volume of distribution of creatinine 1 day after transplantation (T1) ${ }^{\mathrm{a}}$

\begin{tabular}{llll}
\hline & Estimate (1) & $\begin{array}{l}\mathrm{D} 3 \mathrm{C}(\mathrm{ml} / \\
\left.\min / 1.73 \mathrm{~m}^{2}\right)^{\mathrm{b}}\end{array}$ & $\begin{array}{l}\text { Difference }(\mathrm{ml} / \\
\left.\min / 1.73 \mathrm{~m}^{2}\right)^{\mathrm{a}}\end{array}$ \\
\hline $\mathrm{Vd}(50 \%$ of BW) & $38.3 \pm 7.6$ & $37.2 \pm 19.8$ & $-4.7 \pm 2.9$ \\
$\mathrm{Vd}(60 \%$ of BW) & $46.0 \pm 9.1$ & $41.9 \pm 22.3$ & $\operatorname{Ref}$ \\
$\mathrm{Vd}(70 \%$ of BW) & $53.7 \pm 10.7$ & $46.7 \pm 24.8$ & $4.7 \pm 2.9$ \\
\hline
\end{tabular}

$D 3 C$ dynamic creatinine clearance calculation, $V d$ volume of distribution, $B W$ bodyweight

${ }^{a}$ Data are expressed as mean $\pm \mathrm{SD}$

${ }^{b} \mathrm{D} 3 \mathrm{C}$ calculated using the different assumptions for the volume of distribution

have a small therapeutic window. In this study, we showed that the D3C has high accuracy in estimating underlying renal function in patients directly after RTX when serum creatinine levels are not in steady-state. In our population, the $\mathrm{D} 3 \mathrm{C}$ showed the best performance compared with the KeGFR and Jelliffe formulas, two previously published DRFFs. Using the D3C early after transplantation may provide nephrologists with an important extra tool to guide drug dosing and possibly identify patients with early renal function decline.

The accuracy of D3C to estimate GFR immediately after RTX was high. Kidney Disease Improving Global Outcomes (KDIGO) guidelines state that the performance of estimated renal function should be measured by the fraction 
of estimates that fall within $30 \%$ of the true value $\left(p_{30 \%}\right)$ [21]. For the D3C, $p_{30 \%}$ was superior to existing DRFFs. This performance was comparable with the performance of widely used MDRD or CKD-EPI formulas to measure GFR (i.e. iohexol clearance) in case of steady-state creatinine [22]. Furthermore, the accuracy (RMSE) of the D3C was slightly higher than that of serial CKD-EPI measurements, suggesting that part of the inaccuracy came from natural fluctuations in GFR over a 2-day period.

Although the three DRFFs are based on the same kinetic principles, there are some crucial differences. In the D3C formula, creatinine production is based on the Cockcroft-Gault formula, which has good accuracy in RTX patients [23]. Studies have shown that the volume of distribution of creatinine and the total body water directly after transplantation on average equals $60 \%$ of total bodyweight, the estimate that is used in the D3C [15-17]. Furthermore, transformation of the D3C to eGFR (in $\mathrm{ml} / \mathrm{min} / 1.73 \mathrm{~m}^{2}$ ) cancels out weight in the formula and makes it comparable to the widely used CKD-EPI formula. The KeGFR provides freedom to use any existing formula of eGFR/eCrCl to calculate creatinine production by multiplying eGFR $(\mathrm{ml} /$ $\left.\mathrm{min} / 1.73 \mathrm{~m}^{2}\right)$ or eCrCl $(\mathrm{ml} / \mathrm{min})$ by the serum creatinine. This method might introduce bias in estimating creatinine production when the eGFR (in $\mathrm{ml} / \mathrm{min} / 1.73 \mathrm{~m}^{2}$ ) deviates from the creatinine clearance (in $\mathrm{ml} / \mathrm{min}$ ). In addition, interpreting $\mathrm{eCrCl}$ as eGFR may lead to overestimation, since creatinine clearance is generally higher than the glomerular filtration rate [23]. Finally, in the KeGFR, the volume of distribution is calculated from the maximum serum creatinine increase per day in an anuric patient, which is often not available. The default value of $132.6 \mu \mathrm{Mol} / \mathrm{l} /$ day for maximum increase is based on reports from the literature, but has not been validated. The Jelliffe formula is based on the creatinine production formula of Jelliffe, and estimates volume of distribution as $40 \%$ of bodyweight. Underestimation of the Jelliffe formula shown in our study is most likely explained by the low estimate of volume of distribution.

Since the D3C is based on standard pharmacokinetic principles that are not restricted to transplantation, it may also be used in other clinical settings. Patients with elevated serum creatinine and suspected acute kidney injury may benefit from a second creatinine measurement and calculation of the D3C to help medication dosing and identify severe acute kidney injury. Indeed, studies have shown that the KeGFR accurately differentiated between protracted acute kidney injury and early recovery in the ICU [10, 24]. Our study adds that in these situations, the D3C may also be used to prevent medication toxicity, which is an important and modifiable consequence of acute kidney injury. Whether the estimates for volume of distribution of the $\mathrm{D} 3 \mathrm{C}$ are also valid in these populations should be investigated, although our analysis showed that the formula is relatively robust to changes in body water composition.

In regard to the results of our study, some limitations should be noted. First, we assumed that the underlying renal function at T1 was the same at T3, although the hemodynamic effects of anesthesia, ischemia-reperfusion injury, and drug effects could have modulated underlying renal function early after transplantation [12, 25]. However, it is likely that these effects would contribute to more variability in the estimated renal function instead of systematic bias over a 2-day period. Moreover, the fact that formulas based on kinetic modeling adequately predict renal eGFR at T3, and the D3C had minimal bias and good accuracy in relation to 24-h urine creatinine clearance at $\mathrm{T} 1$, favors the assumption that the underlying renal function at $\mathrm{T} 1$ is a robust predictor of eGFR at T3. Including urinary parameters such as creatinine excretion in the formula would theoretically allow more detailed evaluation of the formula. However, it is of note that urine collection is prone to error, which favors the estimation of renal function using DRFFs, without the use of urine collection [3]. Finally, our formula quantifies total renal function and does not distinguish between residual renal function and graft function.

\section{Conclusion}

The D3C is a new DRFF in RTX patients that enables accurate assessment of renal function early after RTX when serum creatinine concentrations are still decreasing. The high accuracy and precision of the $\mathrm{D} 3 \mathrm{C}$ allows close monitoring of the eGFR immediately after RTX, and optimal dosing of potential toxic drugs that depend on renal clearance in a vulnerable patient population.

\section{Compliance with Ethical Standards}

Funding No external funding was used in the preparation of this manuscript.

Conflicts of interest Tobias T. Pieters, Paul Beele, Arjan D. Van Zuilen, Marianne C. Verhaar, Alwin D.R. Huitema, and Maarten B. Rookmaaker declare they have no potential conflicts of interest that might be relevant to the contents of this manuscript.

Ethical approval This study was performed according to the declaration of Helsinki and the ethical guidelines of our institution. The clinical and research activities being reported are consistent with the Principles of the Declaration of Istanbul as outlined in the Declaration of Istanbul on Organ Trafficking and Transplant Tourism. Anonymized clinical data were gathered via our transplant database. Acquiring additional informed consent or official approval of the ethical board was not required, as was confirmed by the ethical board of our hospital. 
Open Access This article is licensed under a Creative Commons Attribution-NonCommercial 4.0 International License, which permits any non-commercial use, sharing, adaptation, distribution and reproduction in any medium or format, as long as you give appropriate credit to the original author(s) and the source, provide a link to the Creative Commons licence, and indicate if changes were made. The images or other third party material in this article are included in the article's Creative Commons licence, unless indicated otherwise in a credit line to the material. If material is not included in the article's Creative Commons licence and your intended use is not permitted by statutory regulation or exceeds the permitted use, you will need to obtain permission directly from the copyright holder.To view a copy of this licence, visit http://creativecommons.org/licenses/by-nc/4.0/.

\section{References}

1. Levey AS, Bosch JP, Lewis JB, Greene T, Rogers N, Roth D. A more accurate method to estimate glomerular filtration rate from serum creatinine: a new prediction equation. Modification of Diet in Renal Disease Study Group. Ann Intern Med. 1999;130(6):461-70.

2. Levey AS, Stevens LA, Schmid CH, Zhang YL, Castro AF 3rd, Feldman HI, et al. A new equation to estimate glomerular filtration rate. Ann Intern Med. 2009;150(9):604-12.

3. O'Sullivan ED, Doyle A. The clinical utility of kinetic glomerular filtration rate. Clin Kidney J. 2017;10(2):202-8. https://doi. org/10.1093/ckj/sfw108.

4. Bragadottir G, Redfors B, Ricksten SE. Assessing glomerular filtration rate (GFR) in critically ill patients with acute kidney injury: true GFR versus urinary creatinine clearance and estimating equations. Crit Care. 2013;17(3):R108. https://doi. org/10.1186/cc12777.

5. Chen S. Retooling the creatinine clearance equation to estimate kinetic GFR when the plasma creatinine is changing acutely. J Am Soc Nephrol. 2013;24(6):877-88. https://doi.org/10.1681/ ASN.2012070653.

6. Jelliffe R. Estimation of creatinine clearance in patients with unstable renal function, without a urine specimen. Am J Nephrol. 2002;22(4):320-4. https://doi.org/10.1159/000065221.

7. Yashiro M, Ochiai M, Fujisawa N, Kadoya Y, Kamata T. Evaluation of estimated creatinine clearance before steady state in acute kidney injury by creatinine kinetics. Clin Exp Nephrol. 2012;16(4):570-9. https://doi.org/10.1007/s10157-012-0602-x.

8. Bouchard J, Macedo E, Soroko S, Chertow GM, Himmelfarb J, Ikizler TA, et al. Comparison of methods for estimating glomerular filtration rate in critically ill patients with acute kidney injury. Nephrol Dial Transplant. 2010;25(1):102-7. https://doi. org/10.1093/ndt/gfp392.

9. Pianta TJ, Endre ZH, Pickering JW, Buckley NA, Peake PW. Kinetic estimation of GFR improves prediction of dialysis and recovery after kidney transplantation. PLoS ONE. 2015;10(5):e0125669. https://doi.org/10.1371/journal.pone.01256 69.

10. de Oliveira MF, Oliveira SA, de Lima ESPF, Nojoza WG, da Silva SM, Ferreira TM, et al. Kinetic estimated glomerular filtration rate in critically ill patients: beyond the acute kidney injury severity classification system. Crit Care. 2017;21(1):280. https://doi. org/10.1186/s13054-017-1873-0.

11. Seelhammer TG, Maile MD, Heung M, Haft JW, Jewell ES, Engoren M. Kinetic estimated glomerular filtration rate and acute kidney injury in cardiac surgery patients. J Crit Care. 2016;31(1):249-54. https://doi.org/10.1016/j.jcrc.2015.11.006.

12. Yarlagadda SG, Coca SG, Garg AX, Doshi M, Poggio E, Marcus RJ, et al. Marked variation in the definition and diagnosis of delayed graft function: a systematic review. Nephrol Dial Transplant. 2008;23(9):2995-3003.

13. Fraser CG. Reference change values. Clin Chem Lab Med. 2011;50(5):807-12. https://doi.org/10.1515/CCLM.2011.733.

14. Siedlecki A, Irish W, Brennan DC. Delayed graft function in the kidney transplant. Am J Transplant. 2011;11(11):2279-96. https ://doi.org/10.1111/j.1600-6143.2011.03754.x.

15. Jones JD, Burnett PC. Creatinine metabolism in humans with decreased renal function: creatinine deficit. Clin Chem. 1974;20(9):1204-12.

16. Chow MS. A method for determining the pharmacokinetics of endogenous creatinine without exogenous creatinine administration. Biopharm Drug Dispos. 1985;6(2):201-8.

17. Coroas A, Oliveira J, Sampaio S, Borges C, Tavares I, Pestana M, et al. Sequential body composition analysis by bioimpedance early post-kidney transplantation. Transpl Int. 2005;18(5):541-7. https ://doi.org/10.1111/j.1432-2277.2005.00086.x.

18. Cockcroft DW, Gault MH. Prediction of creatinine clearance from serum creatinine. Nephron. 1976;16(1):31-41. https://doi. org/10.1159/000180580.

19. Nguyen MT, Maynard SE, Kimmel PL. Misapplications of commonly used kidney equations: renal physiology in practice. Clin J Am Soc Nephrol. 2009;4(3):528-34. https://doi.org/10.2215/ CJN.05731108

20. Du Bois D, Du Bois EF. A formula to estimate the approximate surface area if height and weight be known. 1916. Nutrition. 1989;5(5):303-11.

21. National Kidney Foundation. K/DOQI clinical practice guidelines for chronic kidney disease: evaluation, classification, and stratification. Am J Kidney Dis. 2002;39(2 Suppl 1):S1-266.

22. Earley A, Miskulin D, Lamb EJ, Levey AS, Uhlig K. Estimating equations for glomerular filtration rate in the era of creatinine standardization: a systematic review. Ann Intern Med. 2012. https ://doi.org/10.7326/0003-4819-156-6-201203200-00391.

23. White CA, Huang D, Akbari A, Garland J, Knoll GA. Performance of creatinine-based estimates of GFR in kidney transplant recipients: a systematic review. Am J Kidney Dis. 2008;51(6):1005-15. https://doi.org/10.1053/j.ajkd.2008.02.308.

24. Dewitte A, Joannes-Boyau O, Sidobre C, Fleureau C, Bats ML, Derache P, et al. Kinetic eGFR and novel AKI biomarkers to predict renal recovery. Clin J Am Soc Nephrol. 2015;10(11):1900 10. https://doi.org/10.2215/CJN.12651214.

25. Nankivell BJ, P'Ng CH, O’Connell PJ, Chapman JR. Calcineurin inhibitor nephrotoxicity through the lens of longitudinal histology: comparison of cyclosporine and tacrolimus eras. Transplantation. 2016;100(8):1723-31. https://doi.org/10.1097/TP.0000000000 001243 . 